\title{
Diseño de sostenimiento dinámico para mitigar los estallidos de rocas
}

\author{
Dynamic support design to mitigate rock burst
}

\author{
Elvis William Valencia Chávez ${ }^{1}$, Enrique Guadalupe Gómez ${ }^{2}$ \\ Recibido: 07/01/2021 - Aprobado: 14/01/2021 - Publicado: 18/06/2021
}

\begin{abstract}
RESUMEN
En más de 100 años de explotación subterránea, se ha profundizado la mina, haciendo aberturas cada vez más grandes por la mecanización, cambiando las condiciones naturales del macizo rocoso, creando problemas de liberación de energía conocidos como estallido de rocas.

La metodología de investigación es cuantitativo, descriptivo y explicativo. Aplicando técnicas de observación se vio la deformación de rocas, se hizo clasificación y pruebas de laboratorio de mecánica de rocas, comportamiento de las ondas sísmicas y otros, determinando las propiedades dinámicas de las rocas. Los resultados indican que la probabilidad de estallidos de roca es de débil a moderado, la resistencia de la roca intacta está en el rango de 92.5 a $163.7 \mathrm{MPa}$, el Squeezing (apretamiento) será de ligero a moderado y otros. El sostenimiento en la mayoría de minas es de tipo estático. El sostenimiento estático alcanza una deformación de hasta 16\% por cada $200.0 \mathrm{~mm}$ de longitud con máxima absorción de energía de 3 $\mathrm{KN} / \mathrm{m}^{2}$, mientras que el sostenimiento dinámico posee una deformación mayor a $600 \mathrm{~mm}$ con una absorción de energía de hasta $40 \mathrm{KN} / \mathrm{m}^{2}$, por ello, se demuestra que esta es la mejor alternativa de sostenimiento para la minería.
\end{abstract}

Palabra clave: Estallidos de roca; sostenimiento dinámico.

\begin{abstract}
In more than 100 years of underground mining, the mine has deepened, making larger openings by mechanization, changing the natural conditions of the rock mass, creating energy release problems known as rock bursting.

The research methodology is quantitative, descriptive and explicatory. By applying observation techniques, the deformation of rocks was seen, classification and laboratory tests of rock mechanics, behavior of seismic waves and others were made, determining the dynamic properties of the rocks. The results indicate that the probability of rock bursting is weak to moderate, the strength of the intact rock is in the range of 92.5 to $163.7 \mathrm{MPa}$, the squeezing will be light to moderate and others. The support in most mines is static. Static support reaches a deformation of up to $16 \%$ for every $200.0 \mathrm{~mm}$ of length with a maximum energy absorption of $3 \mathrm{KN} / \mathrm{m}^{2}$, while dynamic support has a deformation greater than $600 \mathrm{~mm}$ with an energy absorption of up to $40 \mathrm{KN} / \mathrm{m}^{2}$. for this reason, it is shown that this is the best alternative to support for mining.
\end{abstract}

Keyword: Rock burst; dynamic support; dynamic bolt.

\footnotetext{
1 Universidad Nacional Mayor de San Marcos, Facultad de Ingeniería Geológica, Minera, Metalúrgica y Geográfica, Departamento Académico de Ingeniería de Minas. Lima, Perú. E-mail: evalencia204@gmail.com - ORCID: https://orcid.org/0000-0003-4981-995X

2 Universidad Nacional Mayor de San Marcos, Facultad de Ingeniería Geológica, Minera, Metalúrgica y Geográfica. Lima, Perú. E-mail: eguadalupeg@unmsm.edu.pe - ORCID: https://orcid.org/0000-0001-9583-8807
} 


\section{INTRODUCCION}

La problemática a resolver era como realizar un sostenimiento dinámico, para mitigar los estallidos de roca, ya que es un tema recurrente en algunas minas del país.

La mina en estudio, se encuentra ubicada en la región Lima norte, se ubica en la vertiente occidental de los andes, en el área del distrito y provincia de Oyón, en el departamento de Lima (Figura 1).

Geológicamente las rocas predominantes corresponden a las calizas de la Formación Jumasha del Cretáceo Superior (Benavides-Caceres, 1956) que corresponden a un metalotecto muy importante. Esta unidad se extiende desde Cerro de Pasco hasta Cajamarca, una posterior inyección magmática produjo venas de cuarzo en calizas (Figura 2), la resistencia compresiva de la caliza es del orden de 60 a $80 \mathrm{MPa}$, pero las calizas con venas de cuarzo llegan a tener una resistencia de 110 a $130 \mathrm{MPa}$. La caliza al ser una roca sedimentaria es dúctil, pero la caliza con venas de cuarzo se vuelve frágil y tiene una baja deformación lo cual lo hace más resistente y es allí donde se produce una acumulación y posterior liberación de energía (estallido de rocas).

\section{MÉTODOS}

La metodología de investigación es cuantitativo, descriptivo y explicativo. Usamos diferentes técnicas de investigación como el cartografiado geomecánico, luego se hizo una clasificación geomecánica de Bieniawski (1989), se determinó la energía potencial de deformación de Kwasniewski, luego se aplicó diversos criterios como el de Wang, Deere y Barla, también se determinó la velocidad de propagación de ondas en roca mediante el equipo Pundit Lab de Proceq, las propiedades dinámicas de rocas en función de ensayos de laboratorio ( $\mathrm{Vp}$ ), monitoreo micro sísmico en función de medición de liberación de energía y finalmente se determinó el sostenimiento dinámico en función de su deformación y así mitigar los estallidos de roca.

\section{RESULTADOS Y DISCUSIÓN}

\subsection{Plano Geomecánico}

En el plano geomecánico del Nv. 3710 en planta (Figura 3), se ubican las cuatro galerías GL6594-1NE; GL6756-1SW; GL6771-SW y GL6980-NE, donde se tiene reportes de presencia de altos esfuerzos según registros del sistema de monitoreo micro sísmico instalado en mina y evaluados por el área de geomecánica, en dicho plano se puede observar la clasificación del macizo rocoso según Bieniawski, donde se determinó que el color amarillo está en el rango de $\mathrm{RMR}=44$ a 48 al cual le corresponde la calidad de macizo roco tipo III y la de color rojo que esta entre el rango de $\mathrm{RMR}=36$ a 41 al cual le corresponde la calidad de macizo rocoso tipo 3 y 4 .

Del evento suscitado en la GL6980-NE y con la información registrada en el sistema de monitoreo micro sísmico se evidencia que el evento sísmico se generó a 203 metros aproximadamente, en la roca caja SE (Figura 4), posterior a ello se ingresó con un equipo multidisciplinario a inspeccionar los diferentes niveles de la zona baja de mina Socorro, tomando la decisión en conjunto de paralizar todas las labores afectada por el evento sísmico, por un plazo de 24 horas, para ser nuevamente evaluadas e iniciar con los trabajos de rehabilitación el cual consistió en realizar el percutado y sostenimiento mecanizado.

En el SNv 6980 -2SW del Nv. 3710 (Figura 5), en el acceso a zona de limpieza Bench and fill, se presenta

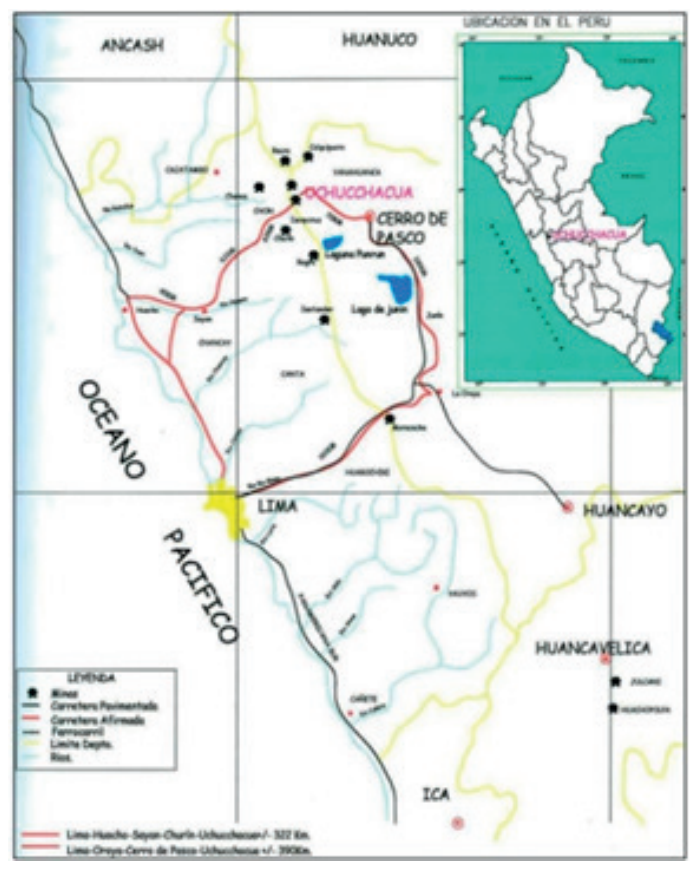

Figura 1. Plano de ubicación

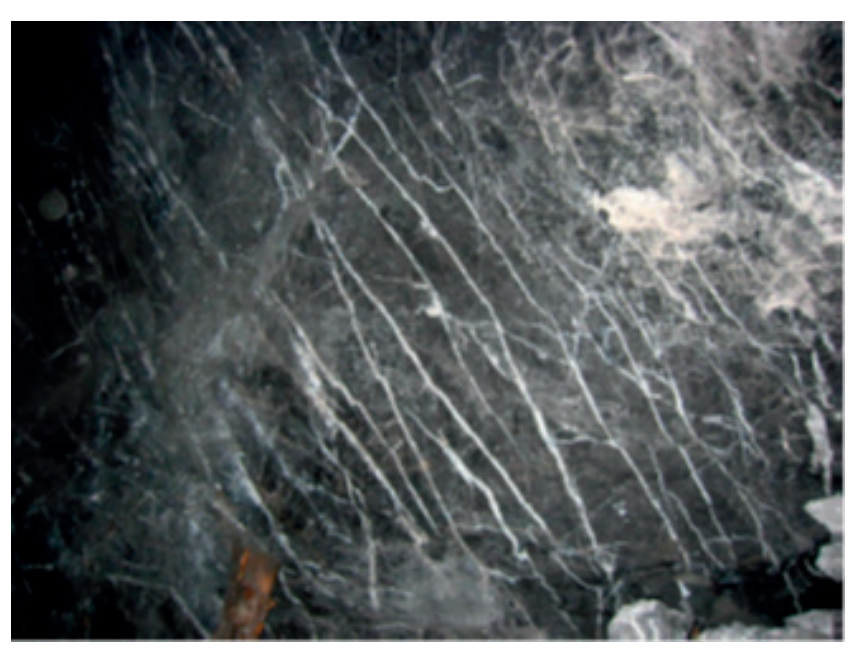

Figura 2. Caliza con venas de cuarzo 
desprendimiento de rocas en la corona y hastiales, producto de ello se presentan rocas en forma de lajas, lo que indica que la roca no pudo deformarse ante la presencia de los esfuerzos inducidos y la masa rocosa se comportó como material frágil.

En el SNv 6980 -3SW del Nv. 3710 (Figura 6), en la zona de acceso de perforación de Bench and fill, se presenta un daño al sostenimiento con rotura de malla electro soldada en una distancia de 20 metros en la corona y hastiales, este tipo de elemento de sostenimiento es rígido dado que son alambres soldados y ante la presencia de esfuerzos inducidos mayor a la resistencia de la masa rocosa, esta se deforma inicialmente y luego colapsa, para estos casos se sugiere usar malla eslabonada.

En la galería 6890 del Nv. 3710 (Figura 7), se puede notar daño al sostenimiento (shotcrete), en una distancia aproximada de 87 metros, la cual se tendrá que rehabilitar, dado que el shotcrete es un elemento de sostenimiento rígido, cuando se presentan esfuerzos inducidos de magnitud mayor a la resistencia del shotcrete esta falla por tracción.

Es conocido que los elementos de sostenimiento como la malla electro soldada y los pernos de roca (Split set, barra helicoidal, inflable, etc.), tienen bajo nivel de deformación que están entre $7 \%$ a $16 \%$ de deformación en longitudes de $200 \mathrm{~mm}$.

\subsection{Clasificación geomecánica según Bieniawski}

Para la clasificación del macizo rocoso se utilizó la clasificación geomecánica de Bieniawski, el cual considera cinco parámetros que son: la resistencia compresiva de la roca intacta, el promedio del espaciado del sistema de fracturas dominante, el grado de fracturamiento RQD de Deere, las condiciones de la masa rocosa (la persistencia de la fractura, apertura de la fractura, rugosidad de las caras de la fractura, el relleno que se encuentra en la fractura y el grado de alteración que sufre la masa rocosa) y la presencia de agua subterránea, de aquí saldrá el valor del RMR básico (solo cuando se requiere conocer únicamente la calidad de la masa roca pero no se va hacer ningún trabajo en ella).

Adicionalmente a ella se requiere hacer un ajuste por orientación en relación al sistema dominante.

De las hojas de mapeo geomecánico (Tablas 1, 2, 3 y 4) del Nv. 3710, se tiene las siguientes características:

G1. 6980 NE. El RMR básico $=44$. Pero al hacer el ajuste $(-5)$ por orientación el $\mathrm{RMR}=39$, al cual le corresponde una calidad de roca tipo IV.

G1. 6594-1 NE. El RMR básico $=54$. Pero al hacer el ajuste (-6) por orientación el $\mathrm{RMR}=48$, al cual le corresponde una calidad de roca tipo III.

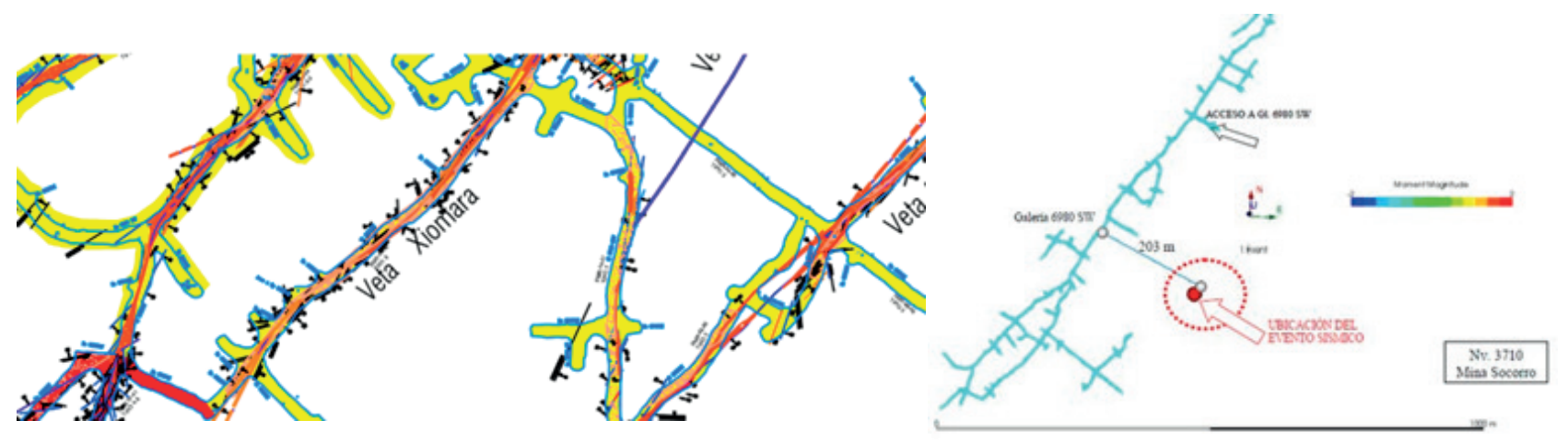

Figura 3. Plano geomecánico del Nv. 3710

Figura 4. Ubicación de evento sísmico del Nv. 3710

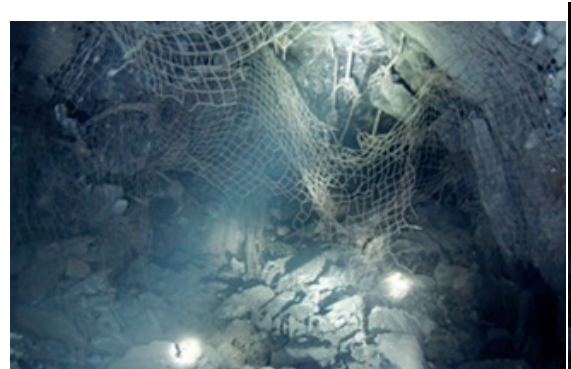

Figura 5. Desprendimiento de rocas en hastiales y corona

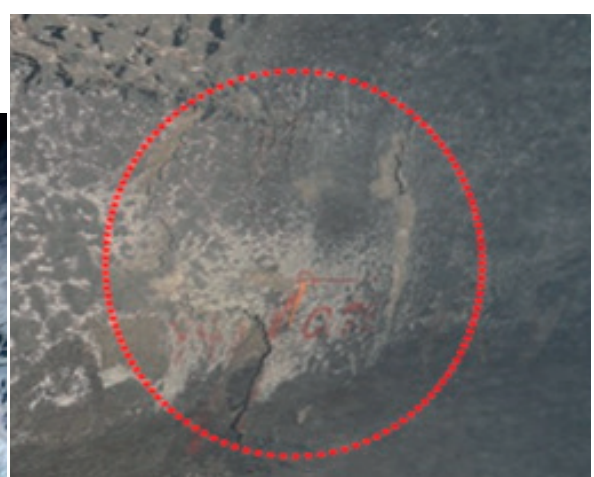

Figura 7. Daño al shotcretre
Figura 6. Daño al sostenimiento en corona y

hastilaes

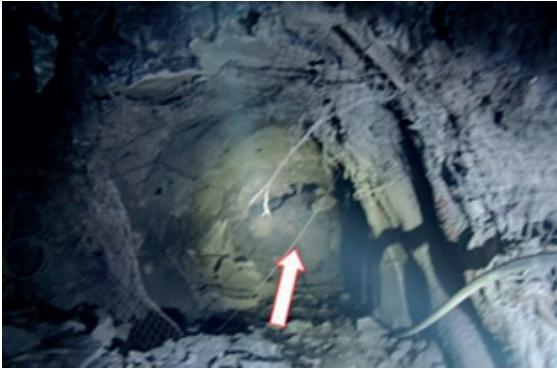


Tabla 1. Mapeo Geomecánico Gl. 6980 NE

\begin{tabular}{|c|c|c|c|c|c|c|c|c|c|c|c|c|c|c|c|}
\hline \multicolumn{16}{|c|}{ Valoración del Macizo Rocoso (R.M.R.) } \\
\hline Nv. 3710 & Socorro Bajo & \multicolumn{14}{|c|}{ GL 6980 NE (3.5X3.5) } \\
\hline \multirow{2}{*}{\multicolumn{2}{|c|}{ Parámetro }} & \multirow{2}{*}{\multicolumn{12}{|c|}{\begin{tabular}{|l} 
Rango de Valores \\
Valor Estimado
\end{tabular}}} & \multicolumn{2}{|c|}{ Valoración } \\
\hline & & & & & & & & & & & & & & & \\
\hline \multicolumn{2}{|c|}{ R. Compre. Uniaxial (MPa) } & $>250$ & (15) & & $100-250$ & (12) & $x$ & $50-100$ & (7) & & $25-50$ & (4) & $<25(2)<5(1) \quad<1(0)$ & 1 & 7 \\
\hline \multicolumn{2}{|l|}{ RQD \% } & $90-100$ & (20) & & $75-90$ & (17) & & $50-75$ & (13) & $\mathrm{x}$ & $25-50$ & (8) & $<25$ & 2 & 8 \\
\hline \multicolumn{2}{|c|}{ Espaciamiento $(\mathrm{m})$} & $>2$ & (20) & & $0,6-2$ & (15) & & $0.2-0.6$ & (10) & $x$ & $0.06-0.2$ & (8) & $<0.06$ & 3 & 8 \\
\hline \multirow{5}{*}{$\begin{array}{l}\text { Condición } \\
\text { de } \\
\text { Juntas }\end{array}$} & Persistencia & $<1 \mathrm{~m}$ long. & (6) & & 1-3 m Long. & (4) & $x$ & $3-10 m$ & (2) & & $10-20 m$ & (1) & $>20 \mathrm{~m}$ & $4 \mathrm{~A}$ & 2 \\
\hline & Abertura & Cerrada & (6) & $\mathrm{x}$ & $<0.1 \mathrm{~mm}$ apert. & (5) & & $0.1-1.0 \mathrm{~mm}$ & (4) & & $1-5 \mathrm{~mm}$ & (1) & $>5 \mathrm{~mm}$ & $4 \mathrm{~B}$ & 5 \\
\hline & Rugosidad & Muy rugosa & (6) & & Rugosa & (5) & $x$ & Lig.rugosa & (3) & & Lisa & (1) & Espejo de falla $(0)$ & $4 \mathrm{C}$ & 3 \\
\hline & Relleno & Limpia & (6) & $x$ & Duro $<5 \mathrm{~mm}$ & (4) & & Duro $>5 \mathrm{~mm}$ & (2) & & Suave $<5 \mathrm{~mm}(1)$ & (1) & Suave $>5 \mathrm{~mm} \quad(0)$ & $4 \mathrm{D}$ & 4 \\
\hline & Alteración & Sana & (6) & & Lig. Alterada. & (5) & $x$ & Mod.Alterada. & (3) & & Muy Alterada. (2) & (2) & Descompuesta $(0)$ & $4 \mathrm{E}$ & 3 \\
\hline \multicolumn{2}{|c|}{ Agua subterránea } & Seco & (15) & & Húmedo & (10) & & Mojado & (7) & $x$ & Goteo & (4) & Flujo & 5 & 4 \\
\hline \multicolumn{14}{|c|}{ Valor Total RMR (Suma de Valoración de 1 al 5 ) } & & 44 \\
\hline \multicolumn{14}{|c|}{ Clase de Macizo Rocoso } & \multirow{3}{*}{\multicolumn{2}{|c|}{ III }} \\
\hline \multirow{2}{*}{\multicolumn{2}{|c|}{$\begin{array}{l}\text { RMR } \\
\text { Descripción }\end{array}$}} & \multirow{2}{*}{\multicolumn{2}{|c|}{$\begin{array}{l}100-81 \\
\text { I Muy Buena }\end{array}$}} & \multirow{2}{*}{\multicolumn{3}{|c|}{$80-61$}} & \multicolumn{3}{|c|}{$60-41$} & \multirow{2}{*}{\multicolumn{3}{|c|}{$40-21$}} & $20-0$ & & \\
\hline & & & & & & & & Regular & & & & & Vuy Mala & & \\
\hline
\end{tabular}

Tabla 2. Mapeo Geomecánico Gl. 6594-1 NE

\begin{tabular}{|c|c|c|c|c|c|c|c|c|c|c|c|c|c|c|}
\hline \multicolumn{15}{|c|}{ Valoración del Macizo Rocoso (R.M.R.) } \\
\hline Nv. 3710 & Socorro Bajo & \multicolumn{13}{|c|}{ GL 6594-1 NE (4.0X4.0) } \\
\hline \multirow{2}{*}{\multicolumn{2}{|c|}{ Parámetro }} & \multirow{2}{*}{\multicolumn{11}{|c|}{$\begin{array}{l}\text { Rango de Valores } \\
\text { Valor Estimado }\end{array}$}} & \multicolumn{2}{|c|}{ Valoración } \\
\hline & & & & & & & & & & & & & & \\
\hline \multicolumn{2}{|c|}{$\begin{array}{l}\text { R. Compre. Uniaxial } \\
(\mathrm{MPa})\end{array}$} & $>250$ & (15) & & $100-250$ & (12) & $x$ & $50-100$ & (7) & & $25-50$ & $<25(2)<5(1) \quad<1(0)$ & 1 & 7 \\
\hline \multicolumn{2}{|l|}{$\mathrm{RQD} \%$} & $90-100$ & (20) & & $75-90$ & $(17)$ & $x$ & $50-75$ & (13) & & $25-50$ & $<25$ & 2 & 13 \\
\hline \multicolumn{2}{|c|}{ Espaciamiento (M) } & $>2$ & (20) & & $0,6-2$ & (15) & & $0.2-0.6$ & (10) & $x$ & $0.06-0.2$ & $<0.06$ & 3 & 8 \\
\hline \multirow{5}{*}{$\begin{array}{l}\text { Condición } \\
\text { de } \\
\text { Juntas }\end{array}$} & Persistencia & $<1 \mathrm{~m}$ long. & (6) & & 1-3 m Long. & (4) & $x$ & $3-10 m$ & (2) & & $10-20 m$ & $>20 \mathrm{~m}$ & $4 \mathrm{~A}$ & 2 \\
\hline & Abertura & Cerrada & (6) & & $<0.1 \mathrm{~mm}$ apert. & (5) & $x$ & $0.1-1.0 \mathrm{~mm}$ & (4) & & $1-5 \mathrm{~mm}$ & $>5 \mathrm{~mm}$ & $4 \mathrm{~B}$ & 4 \\
\hline & Rugosidad & Muy rugosa & (6) & & Rugosa & (5) & $x$ & Lig.rugosa & (3) & & Lisa & Espejo de falla & $4 C$ & 3 \\
\hline & Relleno & Limpia & (6) & $x$ & Duro $<5 \mathrm{~mm}$ & (4) & & Duro $>5 \mathrm{~mm}$ & (2) & & Suave $<5 \mathrm{~mm}$ (1) & Suave $>5 \mathrm{~mm} \quad$ (0) & $4 \mathrm{D}$ & 4 \\
\hline & Alteración & Sana & (6) & & Lig. Alterada. & (5) & $x$ & Mod.Alterada. & (3) & & Muy Alterada. (2) & Descompuesta $\quad(0)$ & $4 \mathrm{E}$ & 3 \\
\hline \multicolumn{2}{|c|}{ Agua Subterránea } & Seco & (15) & & Húmedo & (10) & & Mojado & (7) & $x$ & Goteo & Flujo & 5 & 10 \\
\hline \multirow{2}{*}{\multicolumn{13}{|c|}{$\begin{array}{l}\text { Valor Total RMR (Suma de Valoración de } 1 \text { al } 5 \text { ) } \\
\text { Clase de Macizo Rocoso }\end{array}$}} & & 54 \\
\hline & & & & & & & & & & & & & \multirow{3}{*}{\multicolumn{2}{|c|}{ III }} \\
\hline \multicolumn{2}{|c|}{ RMR } & $100-81$ & & & -61 & & & -41 & & & -21 & $20-0$ & & \\
\hline \multicolumn{2}{|l|}{ Descripción } & I Muy Buena & & & Buena & & III & Regular & & & Mala & V Muy Mala & & \\
\hline
\end{tabular}

Tabla 3. Mapeo Geomecánico Gl. 6771 SW

\begin{tabular}{|c|c|c|c|c|c|c|c|c|c|c|c|c|c|c|}
\hline \multicolumn{15}{|c|}{ Valoración del Macizo Rocoso (R.M.R.) } \\
\hline Nv. 3710 & Socorro Bajo & \multicolumn{13}{|c|}{ GL 6771 SW (3.5X3.5) } \\
\hline \multirow{2}{*}{\multicolumn{2}{|c|}{ Parámetro }} & \multirow{2}{*}{\multicolumn{11}{|c|}{\begin{tabular}{|l|} 
Rango de Valores \\
Valor Estimado
\end{tabular}}} & \multicolumn{2}{|c|}{ Valoración } \\
\hline & & & & & & & & & & & & & & \\
\hline \multicolumn{2}{|c|}{ R. Compre. Uniaxial (MPa) } & $>250$ & (15) & & $100-250$ & (12) & $x$ & $50-100$ & (7) & & $25-50$ & $<25(2)<5(1)<1(0)$ & 1 & 7 \\
\hline \multicolumn{2}{|l|}{$\mathrm{RQD} \%$} & $90-100$ & (20) & & $75-90$ & (17) & $x$ & $50-75$ & (13) & & $25-50$ & $<25$ & 2 & 13 \\
\hline \multicolumn{2}{|c|}{ Espaciamiento (m) } & $>2$ & (20) & & $0,6-2$ & $(15)$ & & $0.2-0.6$ & $(10)$ & $x$ & $0.06-0.2$ & $<0.06$ & 3 & 8 \\
\hline \multirow{5}{*}{$\begin{array}{l}\text { Condición } \\
\text { de } \\
\text { Juntas }\end{array}$} & Persistencia & $<1 \mathrm{~m}$ long. & (6) & & 1-3 m Long. & (4) & $x$ & $3-10 m$ & (2) & & $10-20 \mathrm{~m}$ & $>20 \mathrm{~m}$ & $4 \mathrm{~A}$ & 2 \\
\hline & Abertura & Cerrada & (6) & $x$ & $<0.1 \mathrm{~mm}$ apert. & $(5)$ & & $0.1-1.0 \mathrm{~mm}$ & (4) & & $1-5 \mathrm{~mm}$ & $>5 \mathrm{~mm}$ & $4 \mathrm{~B}$ & 5 \\
\hline & Rugosidad & Muy rugosa & (6) & & Rugosa & (5) & $x$ & Lig.rugosa & (3) & & Lisa & Espejo de falla & $4 \mathrm{C}$ & 3 \\
\hline & Relleno & Limpia & (6) & $x$ & Duro $<5 \mathrm{~mm}$ & (4) & & Duro $>5 \mathrm{~mm}$ & (2) & & Suave $<5 \mathrm{~mm}$ (1) & Suave $>5 \mathrm{~mm} \quad(0)$ & $4 \mathrm{D}$ & 4 \\
\hline & Alteración & Sana & (6) & & Lig. Alterada. & (5) & $x$ & Mod.Alterada. & (3) & & Muy Alterada. (2) & Descompuesta (0) & $4 \mathrm{E}$ & 3 \\
\hline \multicolumn{2}{|c|}{ Agua Subterránea } & Seco & (15) & & Húmedo & (10) & & Mojado & (7) & $x$ & Goteo & Flujo & 5 & 10 \\
\hline \multirow{2}{*}{\multicolumn{13}{|c|}{$\begin{array}{l}\text { Valor Total RMR (Suma de Valoración de } 1 \text { al } 5 \text { ) } \\
\text { Clase de Macizo Rocoso }\end{array}$}} & & 55 \\
\hline \multirow{2}{*}{\multicolumn{9}{|c|}{$\begin{array}{l}\text { Clase de Macizo Rocoso } \\
\text { RMR }\end{array}$}} & & & & & \multirow{3}{*}{\multicolumn{2}{|c|}{ III }} \\
\hline RMR & & & & & & & \multicolumn{2}{|c|}{$60-41$} & & \multirow{2}{*}{\multicolumn{2}{|c|}{\begin{tabular}{|l|l|}
$40-21$ \\
IV Mala
\end{tabular}}} & & & \\
\hline \multicolumn{2}{|c|}{ Descripción } & \multicolumn{2}{|l|}{ I Muy Buena } & \multicolumn{3}{|c|}{ II Buena } & IIII & Regular & & & & V Muy Mala & & \\
\hline
\end{tabular}


Tabla 4. Mapeo Geomecánico GI. 6753-1 SW

\begin{tabular}{|c|c|c|c|c|c|c|c|c|c|c|c|c|c|c|}
\hline \multicolumn{15}{|c|}{ Valoración del Macizo Rocoso (R.M.R.) } \\
\hline Nv. 3710 & Socorro Bajo & \multicolumn{13}{|c|}{ GL 6753-1 SW (4.0X4.0) } \\
\hline \multirow{2}{*}{\multicolumn{2}{|c|}{ Parámetro }} & \multirow{2}{*}{\multicolumn{11}{|c|}{$\begin{array}{l}\text { Rango de Valores } \\
\text { Valor Estimado }\end{array}$}} & \multicolumn{2}{|c|}{ Valoración } \\
\hline & & & & & & & & & & & & & & \\
\hline \multicolumn{2}{|c|}{\begin{tabular}{|l|} 
R. Compre. Uniaxial (MPa) \\
\end{tabular}} & $>250$ & $(15)$ & & $100-250$ & $(12)$ & $x$ & $50-100$ & $(7)$ & & $25-50$ & $<25(2)<5(1)<1(0)$ & 1 & 7 \\
\hline \multicolumn{2}{|l|}{\begin{tabular}{|l|} 
RQD $\%$ \\
\end{tabular}} & $90-100$ & (20) & & $75-90$ & (17) & $x$ & $50-75$ & (13) & & $25-50$ & $<25$ & 2 & 13 \\
\hline \multicolumn{2}{|c|}{ Espaciamiento (m) } & $>2$ & (20) & & $0,6-2$ & (15) & & $0.2-0.6$ & (10) & $x$ & $0.06-0.2$ & $<0.06$ & 3 & 8 \\
\hline \multirow{5}{*}{$\begin{array}{l}\text { Condición } \\
\text { de } \\
\text { Juntas }\end{array}$} & Persistencia & $<1 \mathrm{~m}$ long. & (6) & & 1-3 m Long. & (4) & $x$ & $3-10 m$ & (2) & & $10-20 m$ & $>20 \mathrm{~m}$ & $4 \mathrm{~A}$ & 2 \\
\hline & Abertura & Cerrada & (6) & $x$ & $<0.1 \mathrm{~mm}$ apert. & (5) & & $0.1-1.0 \mathrm{~mm}$ & (4) & & $1-5 \mathrm{~mm}$ & $>5 \mathrm{~mm}$ & $4 \mathrm{~B}$ & 5 \\
\hline & Rugosidad & Muy rugosa & (6) & & Rugosa & (5) & $x$ & Lig.rugosa & (3) & & Lisa & Espejo de falla $(0)$ & $4 \mathrm{C}$ & 3 \\
\hline & Relleno & Limpia & (6) & $x$ & Duro $<5 \mathrm{~mm}$ & (4) & & Duro $>5 \mathrm{~mm}$ & (2) & & Suave $<5 \mathrm{~mm}$ (1) & Suave $>5 \mathrm{~mm} \quad(0)$ & $4 \mathrm{D}$ & 4 \\
\hline & Alteración & Sana & (6) & & Lig. Alterada. & (5) & $x$ & Mod.Alterada. & (3) & & Muy Alterada. (2) & Descompuesta $(0)$ & $4 \mathrm{E}$ & 3 \\
\hline \multicolumn{2}{|c|}{ Agua Subterránea } & Seco & (15) & & Húmedo & (10) & & Mojado & $(7)$ & $x$ & Goteo & Flujo & 5 & 10 \\
\hline \multicolumn{13}{|c|}{ Valor Total RMR (Suma de Valoración de 1 al 5 ) } & & 55 \\
\hline \multicolumn{13}{|c|}{ Clase de Macizo Rocoso } & \multirow{3}{*}{\multicolumn{2}{|c|}{ 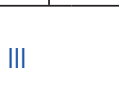 }} \\
\hline \multirow{2}{*}{\multicolumn{2}{|c|}{\begin{tabular}{|l} 
RMR \\
Descripción
\end{tabular}}} & \multirow{2}{*}{\multicolumn{2}{|c|}{\begin{tabular}{|l|}
$100-81$ \\
I Muy Buena \\
\end{tabular}}} & \multirow{2}{*}{\multicolumn{3}{|c|}{$\begin{array}{l}80-61 \\
\text { Buena }\end{array}$}} & \multicolumn{3}{|c|}{$60-41$} & \multicolumn{2}{|c|}{$40-21$} & \multirow{2}{*}{\begin{tabular}{|l|}
$20-0$ \\
V Muy Mala \\
\end{tabular}} & & \\
\hline & & & & & & & & Regular & & & Mala & & & \\
\hline
\end{tabular}

G1. 6771 SW. El RMR básico $=55$. Pero al hacer el ajuste $(-5)$ por orientación el $\mathrm{RMR}=50$, al cual le corresponde una calidad de roca tipo III.

Gl. 6753-1 SW. El RMR básico $=55$. Pero al hacer el ajuste $(-5)$ por orientación el $\mathrm{RMR}=50$, al cual le corresponde una calidad de roca tipo III.

Además, la resistencia compresiva uniáxial de la roca se encuentra en el rango de 50-100 $\mathrm{MPa}$ pero en campo arrojó un valor de resistencia de $85 \mathrm{MPa}$, el espaciamiento promedio del sistema dominante con rango de 0.06 a 0.20 metros obteniéndose bloque pequeños a medianos, el grado de fracturamiento las condiciones de discontinuidad el cual se encuentra en un rango de 50 a $75 \%$ y la presencia de agua subterránea, la misma que fue corregida por orientación, determinándose el RMR ajustado, arrojando un valor promedio de $\mathrm{RMR}=50$. Hay que tener en cuenta que la roca presenta venillas de cuarzo dentro en las cajas, lo cual aumenta su calidad geomecánica (Bieniawski, 1989).

\subsection{Monitoreo micro sísmico}

Se consideró un monitoreo micro sísmico donde se observó diferentes valores máximos de acumulación de energía desde 0.08 Joules hasta 7741501.5 Joules, notándose una gran variación de valores intermedios, los que nos hace suponer que cada uno de ellos responden a una magnitud de momento que pudieron ser causados por el disparo, activación de fallas, secuencia de minado, tipo de roca y profundidad.

El sistema de monitoreo micro sísmico consiste en la observación pasiva de sismos a pequeña escala, con una magnitud menor a cero, los cuales ocurren como resultado de la actividad humana como la minería durante la explotación, fracturación y otros.

El mapeo microsísmico tradicional determina la ubicación y magnitud del evento. Cuando la micro sismicidad es observada a través del tiempo, los operadores pueden empezar a ver patrones de sismicidad relacionados con actividades de producción

\subsection{Determinación de la energía potencial de deformación de Kwasniewski}

Se tomó muestras de roca de los cuatro tajeos considerados GL6594-1NE; GL6756-1SW; GL6771-SW y GL6980NE y se realizó ensayos de compresión simple uniáxial no confinada y constantes elásticas, pero en condiciones servo controladas, esto es con control de carga constante, para determinar la probabilidad de estallido de roca, los resultados del PES indican probabilidad de estallidos moderados a altos (Kwasniewski \& Lydzba, 2013) (Tabla 5).

Tabla 5. Resultados de probabilidad de estallidos de roca según Kwasniewski

\begin{tabular}{ccccc}
\hline Muestra & $\begin{array}{c}\text { Resistencia } \\
\text { (MPA) }\end{array}$ & $\begin{array}{c}\text { M. Young } \\
\text { (GPA) }\end{array}$ & Poisson & $\begin{array}{c}\text { PES } \\
\text { (Kj/m3) }\end{array}$ \\
\hline M-1 & 85,9 & 17,9 & 0,25 & 206,11 \\
M-2 & 75,9 & 21,4 & 0,23 & 134,60 \\
M-3 & 75,4 & 17,8 & 0,24 & 159,70 \\
M-4 & 72,5 & 20,5 & 0,22 & 128,20 \\
M-5 & 65,6 & 27,4 & 0,21 & 78,53 \\
M-16 & 80,7 & 19,4 & 0,25 & 167,85 \\
M-27 & 85,9 & 20,5 & 0,24 & 179,97 \\
M-39 & 86,5 & 21,2 & 0,22 & 176,47 \\
M-42 & 79,3 & 19,7 & 0,25 & 159,61 \\
M-43 & 76,5 & 21,2 & 0,26 & 138,02 \\
\hline
\end{tabular}

\subsection{Criterio de Wang}

Que considera tanto el estado tensional del macizo rocoso como las propiedades mecánicas de las rocas, y constituye una evolución del Criterio de falla de Hoek. Los resultados de cálculo del esfuerzo tangencial considerando una profundidad cercana a los seiscientos cincuenta metros con una densidad promedio de $2.70 \mathrm{gr} / \mathrm{cc}$, los resultados 
muestran una probabilidad de estallidos de roca de débil a moderado (Wang et al., 2021) (Tabla 6).

Tabla 6. Resultados de probabilidad de estallidos de roca según Wang

\begin{tabular}{cccc}
\hline Muestra & $\begin{array}{c}\text { Esf. Tangencial } \\
\text { (MPA) }\end{array}$ & $\begin{array}{c}\text { R. Compresiva } \\
\text { (MPA) }\end{array}$ & Ts \\
\hline M-1 & 22,7 & 85,9 & $\mathbf{0 , 2 6}$ \\
M-2 & 22,7 & 75,9 & $\mathbf{0 , 3 0}$ \\
M-3 & 22,7 & 75,4 & $\mathbf{0 , 3 0}$ \\
M-4 & 22,7 & 72,5 & $\mathbf{0 , 3 1}$ \\
M-5 & 22,7 & 65,6 & $\mathbf{0 , 3 5}$ \\
M-16 & 22,7 & 80,7 & $\mathbf{0 , 2 8}$ \\
M-27 & 22,7 & 85,9 & $\mathbf{0 , 2 6}$ \\
M-39 & 22,7 & 86,5 & $\mathbf{0 , 2 6}$ \\
M-42 & 22,7 & 79,3 & $\mathbf{0 , 2 9}$ \\
M-43 & 22,7 & 76,5 & 0,30 \\
\hline
\end{tabular}

\subsection{Criterio de Deere}

Sobre la calidad de roca según el fracturamiento y de los reportes de la zona problema del Nv.3710 en las galerías 6594-1NE, 6753-1SW, 6771 SW y 6771 NE, el RQD está en el rango de $50 \%$ - 75\% y según indica las tablas 1, 2, 3 y 4. El riesgo de estallidos de rocas es moderado (Deere, 1964).

\subsection{Criterio de Barla}

Mediante el cociente de la resistencia del macizo rocoso entre el producto de la densidad de la roca y la cobertura se podría estimar el grado de squeezing (Barla, 2001).

$$
\frac{\sigma_{\text {cmass }}}{y z}
$$

Según los datos de ensayos de compresión triaxial obtenidos en muestras de campo de las galerías GL65941NE; GL6756-1SW; GL6771-SW y GL6980-NE., (Tabla $7)$, se obtuvo los siguientes valores.

\subsection{Paul Marinos \& Evert Hoek (2018)}

Del artículo Geologically friendly tool for rock mass strength estimation (Marinos \& Hoek, 2018), se ingresa con el GSI correlacionado con el RMR (45) menos cinco unidades, el cual nos da un valor de 40 y el valor del mi obtenido del ensayo de compresión triaxial (Tabla 7), se obtiene la relación del criterio de falla de Hoek \& Brown (1980), que es un gráfico (Figura 8), y se tiene:

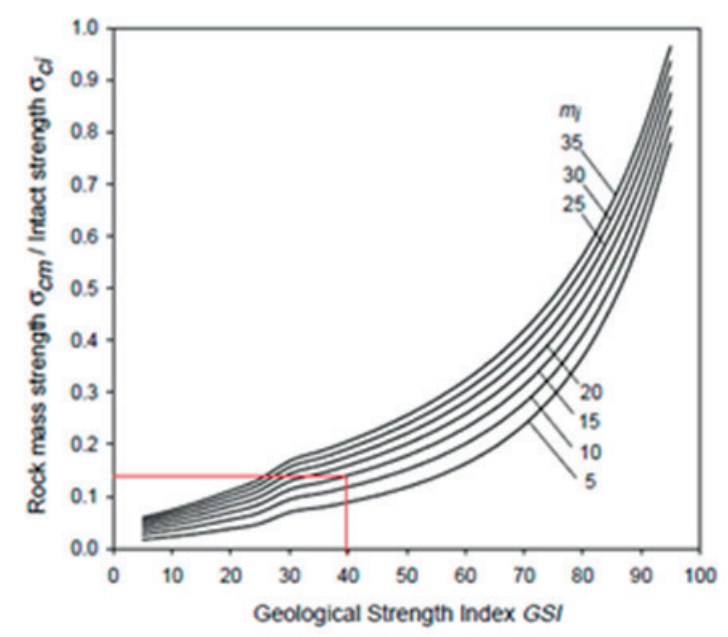

Figura 8. Determinación de la resistencia del macizo rocoso. Fuente: Marinos \& Hoek (2018)

Dónde: $\sigma \mathrm{mr} / \sigma \mathrm{ci}=0.15$

Tabla 7. Resultados de ensayos de compresión Triaxial servo controlado

\begin{tabular}{|c|c|c|c|c|c|c|c|}
\hline Muestra & $\begin{array}{c}\text { Diámetro } \\
\text { (cm) }\end{array}$ & $\begin{array}{l}\text { Altura } \\
(\mathrm{cm})\end{array}$ & $\begin{array}{c}\text { Confinamiento } \\
\sigma_{3} \\
(\mathrm{MPa}) \\
\end{array}$ & $\begin{array}{c}\text { Esfuerzo rotura } \\
\sigma_{1} \\
(\mathrm{MPa}) \\
\end{array}$ & $\begin{array}{c}\text { Constante } \\
\text { "mi" }\end{array}$ & $\begin{array}{c}\text { Cohesión } \\
\text { "c" } \\
\text { (MPa) }\end{array}$ & $\begin{array}{l}\text { Ang. de Fricción } \\
\text { Interno } \\
\left({ }^{\circ}\right)\end{array}$ \\
\hline \multirow{3}{*}{$\mathrm{M}-17$} & 4.76 & 9.38 & 2 & 114.1 & & & \\
\hline & 4.76 & 9.28 & 4 & 126.6 & 12.59 & 20.53 & 46.07 \\
\hline & 4.76 & 9.40 & 6 & 138.7 & & & \\
\hline \multirow{3}{*}{ M-26 / M-25 } & 4.71 & 9.35 & 2 & 114.1 & & & \\
\hline & 4.71 & 9.40 & 4 & 127.6 & 13.40 & 20.01 & 46.94 \\
\hline & 4.71 & 9.41 & 6 & 139.8 & & & \\
\hline \multirow{3}{*}{ M-39 / M-38 } & 4.73 & 9.21 & 2 & 110.8 & & & \\
\hline & 4.73 & 9.26 & 4 & 124.8 & 14.95 & 18.52 & 48.24 \\
\hline & 4.73 & 9.05 & 6 & 138.3 & & & \\
\hline \multirow{3}{*}{ M-42 } & 4.72 & 9.26 & 2 & 92.5 & & & \\
\hline & 4.73 & 9.37 & 4 & 105.8 & 14.40 & 15.65 & 47.09 \\
\hline & 4.73 & 9.31 & 6 & 118.4 & & & \\
\hline \multirow{3}{*}{ M-44 / M-45 } & 4.71 & 9.32 & 2 & 134.1 & & & \\
\hline & 4.71 & 9.35 & 4 & 149.3 & 15.83 & 21.95 & 49.63 \\
\hline & 4.71 & 9.28 & 6 & 163.7 & & & \\
\hline
\end{tabular}


Si tomamos en cuenta que la resistencia promedio de la roca es $105 \mathrm{MPa}$, se deduce que la resistencia del macizo rocoso es 15.75 MPA en promedio.

A partir de ello procedemos a calcular de acuerdo a la teoría de Barla (2001), al reemplazar los valores de la densidad de la roca y la carga litostática, nos arroja un valor de 0.93 , el cual corresponde a un squeezing ligero a moderado.

\subsection{Velocidad de propagación de ondas}

Asimismo, se realizó ensayos de velocidad de propagación de onda con el equipo Pundit Lab+ (Proceq). (Tabla 8), arrojando los siguientes valores:

Tabla 8. Resultados de la velocidad de propagación de ondas con el Pundit Lab+

\begin{tabular}{ccccc}
\hline Muestra & $\begin{array}{c}\text { Distancia } \\
(\mathbf{m})\end{array}$ & $\begin{array}{c}\text { Tiempo } \\
(\boldsymbol{\mu s})\end{array}$ & $\begin{array}{c}\text { Velocidad } \\
(\mathrm{m} / \mathbf{\mu s})\end{array}$ & $\begin{array}{c}\text { Velocidad } \\
(\mathrm{m} / \mathbf{s})\end{array}$ \\
\hline M-17 & 0,0938 & 36,4 & 0,0026 & 2576,9231 \\
M-17 & 0,0928 & 38,2 & 0,0024 & 2429,3194 \\
M-17 & 0,094 & 32,4 & 0,0029 & 2901,2346 \\
M-25 & 0,0935 & 31,6 & 0,0030 & 2958,8608 \\
M-25 & 0,094 & 33,8 & 0,0028 & 2781,0651 \\
M-26 & 0,0941 & 34,5 & 0,0027 & 2727,5362 \\
M-38 & 0,0921 & 39,1 & 0,0024 & 2355,4987 \\
\hline
\end{tabular}

Según los resultados obtenidos de la probabilidad de estallidos de roca determinados por medios empíricos, se concluye que el sostenimiento deberá ser dinámico, esto es el sostenimiento deberá tener alta capacidad de deformación para poder mitigar los altos esfuerzos producto de la secuencia de minado.

\subsection{Resultados de la Pruebas de Pull test del perno dinámico Durabar}

Para poder mitigar la energía liberada según data del monitoreo micro sísmico, se instaló el perno dinámico
Durabar, obteniendo excelentes resultados. (Tabla 9), (Figuras 9.10.11 y 12)

De los resultados obtenidos se puede notar que la deformación del perno Durabar está en el rango de $60.0 \mathrm{~mm}$.

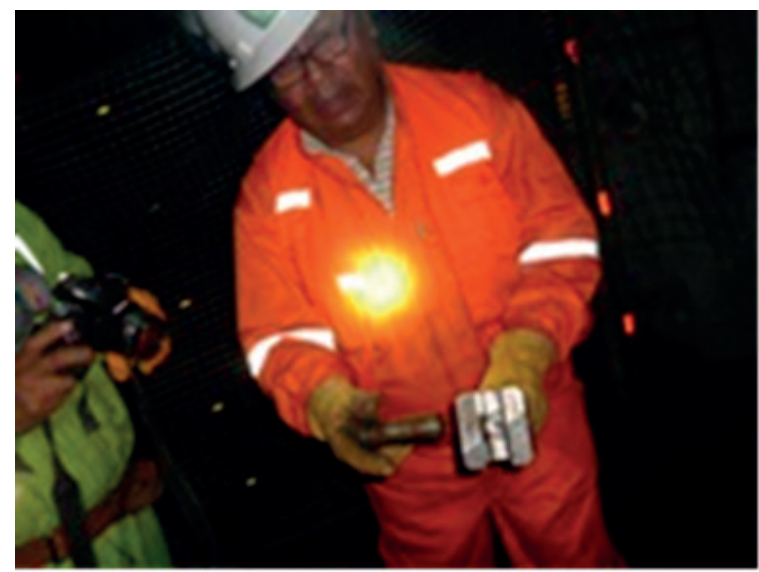

Figura 9. Instalación de garra

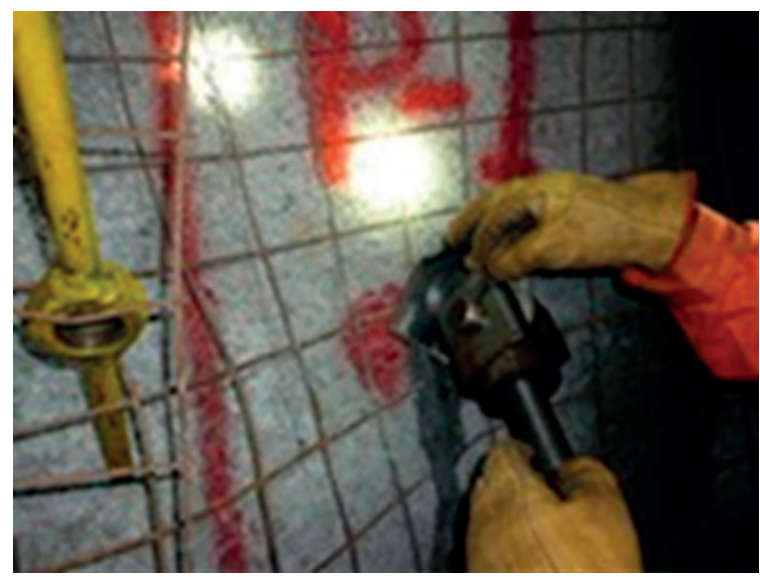

Figura 10. Sujeción del perno

Tabla 9. Pruebas de Pull Test al perno dinámico (Durabar)

\begin{tabular}{|c|c|c|c|c|c|}
\hline $\begin{array}{l}\text { Carga } \\
\text { (Ton) }\end{array}$ & $\begin{array}{c}\text { Desplazamiento } \\
\text { Durabar } 1 \\
(\mathrm{~mm})\end{array}$ & $\begin{array}{c}\text { Desplazamiento } \\
\text { Durabar } 2 \\
(\mathrm{~mm})\end{array}$ & $\begin{array}{c}\text { Desplazamiento } \\
\text { Durabar } 3 \\
(\mathrm{~mm})\end{array}$ & $\begin{array}{c}\text { Desplazamiento } \\
\text { Durabar } 4 \\
(\mathrm{~mm})\end{array}$ & $\begin{array}{c}\text { Desplazamiento } \\
\text { Durabar } 5 \\
(\mathrm{~mm})\end{array}$ \\
\hline 0 & 0 & 0 & 0 & 0 & 0 \\
\hline 4 & 1.5 & 0.5 & 1.3 & 2 & 3.1 \\
\hline 8 & 6.4 & 0.8 & 7 & 8.9 & 7.6 \\
\hline 12 & 15.2 & 0.8 & 10.1 & 15.4 & 7.9 \\
\hline 16 & 18.9 & 1.0 & 14.2 & 21 & 8.4 \\
\hline 20 & 21.3 & 1.1 & 18.3 & 24.8 & 8.9 \\
\hline 24 & 23.1 & 1.1 & 19.4 & 26.3 & 10 \\
\hline 28 & 27.3 & 1.7 & 20.5 & 28.1 & 11.9 \\
\hline 32 & 33.2 & 2.6 & 22.5 & 32.5 & 14.3 \\
\hline 36 & 41.0 & 3.6 & 25.5 & 39.1 & 17.2 \\
\hline 40 & 49.1 & 4.7 & 32.5 & 47.6 & 20.1 \\
\hline 42 & 65.0 & 5.9 & 58.5 & 58.1 & 60.3 \\
\hline
\end{tabular}




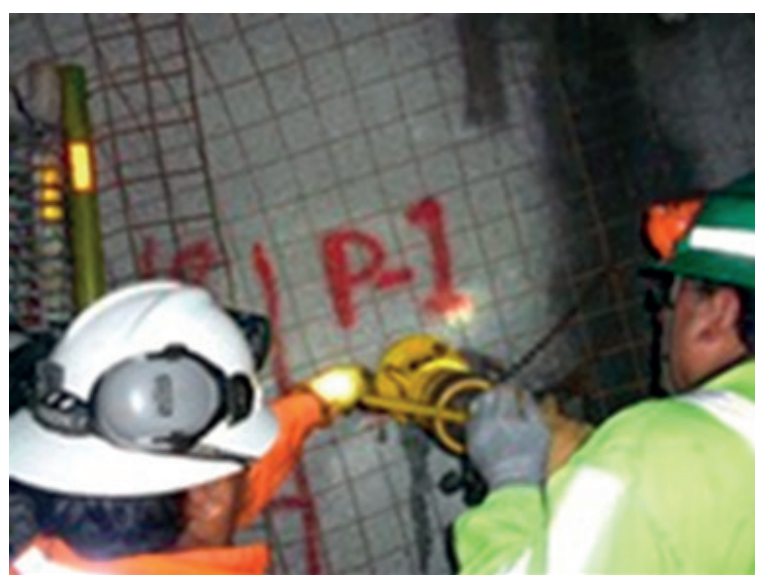

Figura 11. Prueba de Pull Test

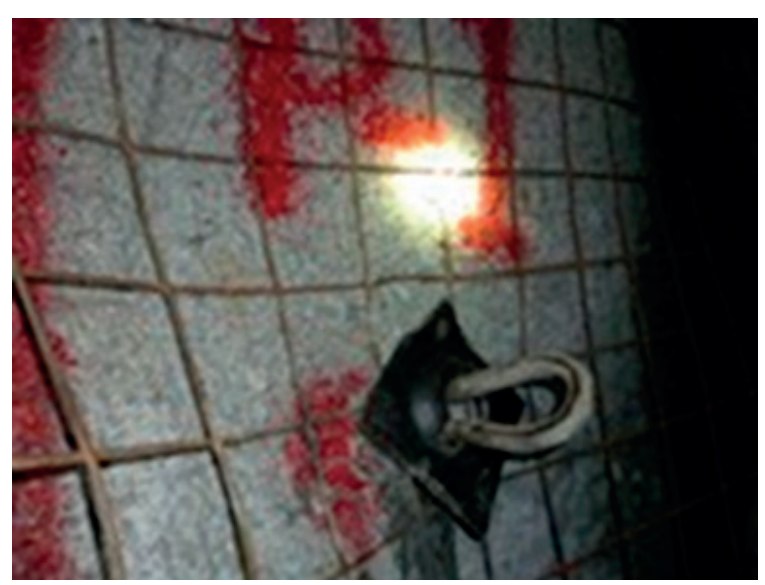

Figura 12. Instalación del perno Durabar

Las minas del Perú solo realizan ensayos mecánicos para un diseño de sostenimiento basado en condición estática, donde estos elementos de sostenimiento tienen poca o ninguna capacidad de absorción de energía, las minas han ido profundizando en estos últimos años lo cual ha traído como consecuencia el incremento de esfuerzos en las operaciones mineras que en algunos casos hacen que el diámetro del taladro disminuya y el perno de roca no pueda ingresar y en otros se presente un evento brusco de liberación de energía.

Una de las condiciones más críticas para la ocurrencia de este evento (estallido de rocas), es la secuencia de minado, por ello se hace necesario instalar sistemas de monitoreo en tiempo real como las instaladas en mina El Porvenir, Uchucchacua, Yauliyacu, otros. Para poder determinar en tiempo real la magnitud de energía acumulada, para tomar decisiones de llevar a buen recaudo al personal y equipos.

Hay que tener en cuenta que son ocho minas las que tienen problemas de altos esfuerzos como, Casapalca, Yauliyacu, El Porvenir, Marsa, Poderosa, Tamboraque, Minsur, Uchucchacua (Berrocal, 2020).

Como discusión de los resultados podemos decir que en la mina Kolar Gold Field de la India el estallido de rocas se produce por la secuencia de minado, la alta resistencia de la roca, fragilidad y poca profundidad (300 m), el mismo que va acompañado de ruido y proyección de roca (Srinivasan et al., 1999). En el caso de mina Socorro el evento se produce por la por la profundidad $(700 \mathrm{~m})$, secuencia de minado y daño al sostenimiento

En caso de mina Casapalca solo se tienen controles de convergencia (extensometría), reportes de operación, instalación de pernos de convergencia Roofex (Barrios, 2009). En mina Socorro se conoce la probabilidad de estallido de rocas, monitoreo micro sísmico, ensayos servo controlado y control de la secuencia de minado en función de la acumulación de energía (Cubas-Castro, 2009).

Se diseñó un sostenimiento flexible según la probabilidad de producirse el estallido de rocas, analizado bajo ensayos de laboratorio servo controlado y determinándose las propiedades dinámicas de la roca en función de la velocidad de propagación de onda y de las magnitudes de liberación de energía, logrando mitigar el estallido de rocas.

\section{CONCLUSIONES}

- En el Nv. 3710. Mina socorro en las galerías GL6594-1NE; GL6756-1SW; GL6771-SW y GL6980-NE, se llevó a cabo el estudio, debido al reporte de eventos sísmicos y falla del sostenimiento, teniendo como resultado de calidad macizo rocoso de tipo III.

- En mina socorro en el Nv. 3710, donde ocurren los eventos sísmicos se encuentra a 700 metros de profundidad, el estallido de roca se produce por la secuencia de minado y la alteración de la roca (venas de cuarzo en caliza), que eleva la calidad geomecánica del macizo rocoso, en mina Oorgaum de Kolar Gold Fields el primer evento sísmico se registró a 300 metros de profundidad, debido a la capacidad de la masa rocosa de almacenar energía (fragilidad de la roca) y por la influencia de esfuerzos remanentes de eventos geológicos pasados. De tal forma que el estallido de rocas en ambas minas se produce por diversos factores, no dependiendo exclusivamente de la profundidad.

- Se diseñó un sostenimiento flexible según la probabilidad de producirse el estallido de rocas y las propiedades dinámicas de la roca, logrando mitigar el estallido de rocas.

- De los ensayos de Pull Test a los pernos dinámicos Durabar 1, 3, 4, y 5 de dos metros de longitud todos ellos presentan una alta deformación (58.1 a $65.0 \mathrm{~mm}$ ) lo cual nos confirma la elasticidad del material con la que está fabricado el perno Durabar, salvo el perno 2 que su deformación fue muy baja $(5.9 \mathrm{~mm})$. Lo cual nos indica que el perno 2 ya estaba activado migando la deformación del macizo rocoso y en el caso de los demás pernos todavía no se presentaban eventos dinámicos y estaban trabajando como pernos estáticos por todo ello, se seleccionó el perno dinámico Durabar por 
su alta elasticidad y absorción de energía para mitigar estallido de rocas.

\section{AGRADECIMIENTOS}

Deseo expresar mi agradecimiento a la FIGMM de la UNI - Laboratorio de Mecánica de Rocas y Rock Lab Ingenieros S.A.C. por brindarme sus instalaciones para realizar los ensayos, estáticos, servo controlados y dinámicos.

\section{REFERENCIAS}

Barla, G. (2001). Tunnelling under squeezing rock conditions Mechanics - Advances in Geotechnical Engineering and Tunnelling, 169-268. https://www.researchgate.net/ publication/259762638

Barrios, J. L. D. (2009). Aplicación de pernos roofex monitor dinámico en la prevención a estallidos de roca mina Casapalca Lima. Compañía Minera Casapalca. https:// es.scribd.com/document/282385445/APLICACION-DEPERNOS-ROOFEX-PREVENCION-ESTALLIDOS-DEROCA1-doc

Benavides-Caceres, V. E. 195. (1956). Cretaceous system in northern Peru. BT - Bulletin of the American Museum of Natural History (Vol. 108, pp. 357-493). http://digitallibrary. amnh.org/handle/2246/1023

Berrocal, M. A. (2020). Mitigación del estallido de roca en excavaciones subterráneas. Revista Seguridad Minera. https://www.revistaseguridadminera.com/operacionesmineras/mitigacion-del-estallido-de-roca-en-excavacionessubterraneas/

Bieniawski, Z. (1989). Rock Mass Classifications. John Wiley \& Sons., 251. https://books.google.com.pe/ books?hl=es\&lr=\&id=pejDUvjwPdMC\&oi=fnd\&pg=PP13 $\& d q=$ Bieniawski, + Z.+T.+(1989).+Engineering + Rock + Ma $\mathrm{ss}+$ Classifications. + Pennsylvania, + USA + John + Wiley $+\%$ $26+$ Sons. + pp. $+45-60 \&$ ots $=4$ UjhSD_-Us\&sig $=z B y W b L 0 c-$ O8ZLk1bn6OGQrgVDeww\#v=onepage\&q\& $\mathrm{f}=\mathrm{fa}$
Cubas-Castro, J. J. (2009). Avances para el control de estallido de rocas mediante el uso de monitoreo microsismico - Mina Uchucchacua. In Repositorio Institucional UNI. http:// cybertesis.uni.edu.pe/handle/uni/11442

Deere, D. U. (1964). Technical description of rock cores for engineering purpose. Rock Mechanics and Engineering Geology, 1(18), 16-22. https://scholar.google.com.pe/ scholar?hl=es\&as $\mathrm{sdt}=0 \% 2 \mathrm{C} 5 \& \mathrm{q}=++$ Deere $\% 2 \mathrm{C}+$ D.U. $\%$ 2C+\% 281963\% $29 \% 2 \mathrm{C}+$ Technical + Description + of + Roc $\mathrm{k}+$ Cores + for + Engineering + Purposes $\% 2 \mathrm{C}+$ Felsmechanik + und + Ingenieur + geologie + Rock + Mechanics + and + Engineer ing + Geology $\% 2 \mathrm{C}+1 \% 3 \mathrm{~A} 1 \% 2 \mathrm{C}+\mathrm{pp} .+16-22 . \& \mathrm{~b}$

Hoek, E., \& Brown, E. T. (1980). Empirical strength criterion for rock masses. Journal of the Geotechnical Engineering Division, ASCE, 106(GT9, Proc. Paper, 15715), 1013-1035. https://doi.org/10.1061/ajgeb6.0001029

Kwasniewski, M., \& Lydzba, D. (2013). Rock Mechanics for Resources, Energy and Environment. In Rock Mechanics for Resources, Energy and Environment. https://doi. org $/ 10.1201 / \mathrm{b} 15683$

Marinos, P., \& Hoek, E. (2018). GSI: A geologically friendly tool for rock mass strength estimation. ISRM International Symposium 2000, IS 2000. https://onepetro. org/ISRMIS/proceedings-abstract/IS00/All-IS00/ISRMIS-2000-035/50905

Srinivasan, C., Arora, S. K., \& Benady, S. (1999). Precursory monitoring of impending rockbursts in Kolar gold mines from microseismic emissions at deeper levels. International Journal of Rock Mechanics and Mining Sciences, 36(7), 941-948. https://doi.org/10.1016/s0148-9062(99)00048-0

Wang, S. ming, Zhou, J., Li, C. qi, Armaghani, D. J., Li, X. bing, \& Mitri, H. S. (2021). Rockburst prediction in hard rock mines developing bagging and boosting tree-based ensemble techniques. Journal of Central South University, 28(2), 527-542. https://doi.org/10.1007/s11771-021-4619-8 
